\title{
MAGNETIC RESONANCE IMAGING VERSUS ARTHROSCOPY FOR TEMPOROMANDIBULAR JOINT INTERNAL DERANGEMENT DIAGNOSIS
}

\author{
Reem H. Hossameldin*
}

\begin{abstract}
Introduction: Temporomandibular joint might be affected by a divergent scope of disorders. The objective of TMJ imaging is to document any clinically suspected disorder of the joint and surrounding structures. MRI has become the gold standard TMJ Imaging. TMJ arthroscopy allow direct visualization of the joint with diagnostic capabilities during surgery

Aim of study: To compare and correlate preoperative TMJ diagnosis based on MRI findings with the perioperative TMJ diagnosis via direct visualization of the same joints during TMJ arthroscopic procedure
\end{abstract}

Materials and methods: 85 patients were selected suffering from internal derangement of TMJ with different Wilkes classification. A total of 108 joints were subjected to first diagnostic arthroscopic TMJ lysis and lavage. Those procedures were done in Department of OMFS, Cairo University, Cairo Egypt.This study was assessed through correlating preoperative TMJ diagnosis based on MRI interpretation, to perioperative TMJ diagnosis based on arthroscopic findings.

Results of investigation: This study revealed that Preoperative diagnosis, based on MRI assessments, came into co-ordinance with arthroscopic findings in $73(67.51 \%)$ studied joints but were different in the remaining $35(32.41 \%)$ joints. Ratios are significantly different between the two modalities of diagnosis, $\mathrm{P}=0.001$. It showed also a statistically significant effect of Wilkes classification on the accuracy of the preoperative MRI diagnosis when compared to the arthroscopic findings.

Conclusion: This study concluded that the primary arthroscopic intervention has a positive effect on having accurate final TMJ diagnosis when compared to preoperative diagnosis based on MRI interpretation which is proved to have limitations.

KEYWORDS: Diagnosis, Temporomandibular, Arthroscopy, MRI, Disorders

\footnotetext{
* Lecturer of Oral \& Maxillofacial Surgery, Faculty of Oral and Dental Medicine, Cairo University, Cairo, Egypt.
} 


\section{INTRODUCTION}

Temporomandibular joint might be affected by a divergent scope of disorders, which could be expressed in some clinical signs and symptoms. The principal objective of TMJ imaging is to document any clinically suspected disorder of the joint and surrounding structures. Multiple imaging modalities are currently available to any clinician evaluating a patient with suspected joint disorder. As TMJ disorders might affect hard and soft components of the joint, any used imaging modality should have the ability to reveal hard components, soft components or both of them. The clinician should select the suitable one after weighting its advantages and limitations. (Boeddinghaus and Whyte 2008). ${ }^{1,2}$ Magnetic resonance imaging (MRI) has become a mainstay in the evaluation of TMJ disorders given its ability of noninvasively depicting major regional anatomic structures and demonstrating the presence of joint effusion and bone marrow signal intensity abnormalities. Consequently, MRI has become the gold standard in diagnosing articular disc displacement. ${ }^{3-7}$ But a point of criticism of MRI of the TMJ is the lack of any ability to assess joint function dynamically as is possible by electronic axiography. ${ }^{8}$ With the advent of computer-driven cine displays (CINE mode) 'dynamic information' should now also be available on MRI. An application of pseudo dynamic MRI was suggested by Conway and Bell. ${ }^{9,10}$ Conway $^{9}$ described how he was able to diagnose a displaced disc and adhesive lesions. For Bell ${ }^{10}$, CINE mode offered the possibility of showing the patient his TMJ dysfunction by video recordings. But there were no urgent reasons for preferring CINE mode. CINE mode can be an additional diagnostic tool in routine static MRI of internal derangement of the TMJ. CINE mode shows only an artificial movement of the disc and condyle of the TMJ and not directly the physiological or pathological situation. Dynamic MRI provides information about disc and condyle mobility, disc reduction and topographic changes in the disc-condyle relationship in various stages of mouth opening. ${ }^{11-14}$ Current literature indicates evidence of the diagnostic accuracy of MRI for evaluating disc position, disc configuration and osseous changes. Still there is insufficient evidence for the diagnostic accuracy and efficacy of MRI to detect TMJ perforation, disc adhesion and joint effusion, and subtle bone marrow changes. ${ }^{15-18}$

One of the diagnostic and surgical management approaches for TMJ diseases is primary TMJ arthroscopy which allows for real time visualization of the superior joint space, as well as the ability to lavage/treat the joint in the same setting. This system allows diagnostic capabilities immediately before and during surgery, ideally maximizing treatment options. ${ }^{17}$ Standardization of TMJ examination procedure was aimed in the Research Diagnostic Criteria for Temporomandibular Disorders (RDC/ TMD) ${ }^{19}$ The value and accuracy of MRI interpretation in TMJ internal derangement diagnosis has been evaluated in small number of studies, addressing the correlation to the clinical findings, as well as surgical findings. ${ }^{20,21}$

\section{AIM OF THE STUDY}

The aim of this study was to compare and correlate preoperative TMJ diagnosis based on MRI findings with the perioperative TMJ diagnosis via direct visualization of the same joints during TMJ arthroscopic procedure.

\section{MATERIALS AND METHODS}

Eighty five patients were selected from the Outpatient clinic of OMFS department of Faculty of Dentistry, Cairo University.

\section{Inclusion criteria consisted of:}

- Clinically reduced mouth opening and/or painful maximum mouth opening.

- Magnetic Resonance Imaging (MRI) demonstration of different Wilkes stages of internal derangement. 
- Unsuccessful non-surgical treatment for at least two months prior to surgery.

\section{Exclusion criteria:}

- Patients with evidence of major jaw trauma.

- Patients with systemic joint or muscle disease: such as rheumatoid arthritis or any other immune arthritis and synovial chondromatosis,...etc.

- Patients with major condylar deformities: Such as avascular condylar necrosis, condylar hyperplasia, condylar osteochondroma, ...etc.

Outcome Variables: preoperative and intraoperative diagnoses were performed on all patients regarding radiographic MRI assessment, and arthroscopic findings, and choosing the Wilkes classification of various internal derangements of TMJ (final staging of internal derangement was reached after performing primary single-puncture arthroscopic procedure).

Arthroscopic lysis and lavage procedure were performed within 1 month of reevaluation after unsuccessful conservative treatment for 2-3 months.

Before the arthroscopic treatment, the patients were examined clinically, based on TMJ assessment standardized form consisting of detailed history and physical examination following the DC/TMD based on the International RDC/TMD Consortium Network RDC/TMD ${ }^{19}$

When indicated and prior to surgical planning. All patients performed TMJ MRI in the following manner.

\section{Magnetic Resonance Imaging (MRI) Evaluation:}

Initial scanning was done to obtain three scout images; one in each plane: coronal, sagittal, and axial. On the sagittal scout view, axial cuts were planned parallel to the orbito-meatal line at the level of auditory canal with the standard parameters
On the axial cuts, a slice was selected on which the condyles of both sides were well depicted. On the selected slice, the sagittal cuts were taken perpendicular on the horizontal long axis of each condyle (parasagittal) and the entire condyles were included inside the planned slices.

\section{»Closed mouth scans:}

Three pulse sequences in the parasagittal plane were taken while the patient was occluding in centric occlusion with the following fixed parameters

\section{The pulse sequences were:}

- Proton density Spin echo PD/SE

- $\quad \mathrm{T} 1$ weighted Spin echo T1/SE

- $\quad \mathrm{T} 2$ weighted Turbo spin echo T2/TSE

\section{Open mouth scan:}

Either proton density or T1 weighted spin echo images were taken according to the better visibility of articular disc in the previously underwent scans. The parameters were similar to those of the closed mouth static scan. Open mouth scan was taken after placing a group of wooden tongue depressors in patient's mouth with their long axis perpendicular to the mid-sagittal plane. The number of tongue depressors was determined before the MRI examination to be suitable for the maximum comfortable mouth opening of each patient.

Based on the clinical and imaging findings, a Wilkes staging was assigned to each joint preoperatively. According to Wilkes stages, the cases were grouped.

\section{Surgical Procedure:}

Under either intra-venous sedation or standard general anesthesia using naso-tracheal intubation, arthroscopic lysis and lavage with mandibular manipulation was performed using a $2.0 \mathrm{~mm}$ miniscope set of $1.9 \mathrm{~mm}$ diameter, 30-degree scope 
(Smith \& Nephew Andover MA, USA). After a single fossa puncture placed in maximum concavity of glenoid fossa. Outflow needle puncture was placed in the following manner: With the mandible protruded, the scope was directed to the center of the fossa area of the joint. The assistant insufflated the joint with $2-3 \mathrm{ml}$ of fluid in order to maintain joint distention. A 22 gauge, 1 1/2 inch needle was inserted approximately $5 \mathrm{~mm}$ anterior and $5 \mathrm{~mm}$ inferior to the fossa puncture site, under joint insufflation. Irrigation system was now patent (Fig. 1). Arthroscopic examination of the superior joint space was performed, and diagnostic sweep was conducted for the seven points of interest which are: medial synovial drape, Pterygoid shadow, retrodiskal synovium, Posterior slope of the articular eminence and glenoid fossa, articular disc, intermediate zone, and anterior recess. With the assessment of Joint dynamics and disc mobility, Arthroscopic assessment of disc position, Arthroscopic, and roofing to specifically grade the amount of displacement by arthroscopic observation of the disc. Then adhesions were detached, and lateral lysis was performed. The mandible was manipulated. The joint space was irrigated with a total of $120 \mathrm{ml}$ of lactated Ringer's solution (LR). After finishing the diagnostic sweep with documentation of anatomical sites and dynamics of the joint, the irrigation was stopped, and lavage needle was removed carefully.

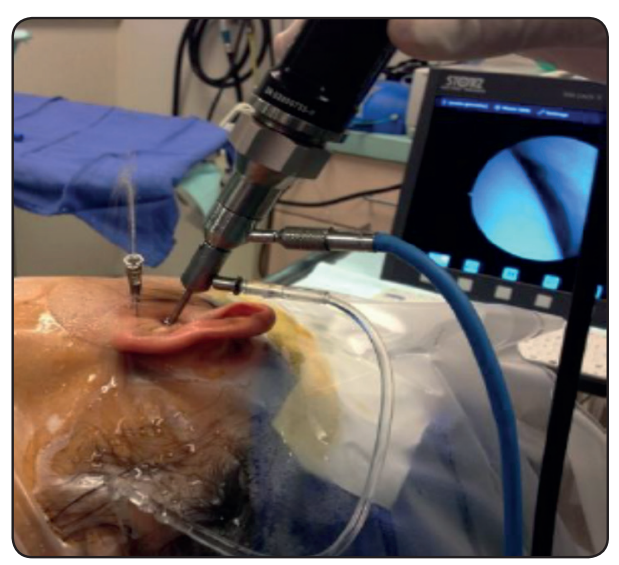

Fig. (1) Establishing a patent outflow lavage track in the joint space

\section{Data Analysis:}

This study was assessed by careful categorization of cases according to different stages of internal derangements, which have been specified by Wilkes classification into five stages according to preoperative clinical and MRI findings. Such findings were correlated to

The intraoperative arthroscopic examination diagnoses.

\section{Statistical Analysis:}

Statistical analysis was performed using SPSS (Statistical package for the social sciences) version 20, IBM corp., USA. Continuous data were represented as means ${ }^{+}$standard deviations where categorical data were represented as frequencies and percentages. Chi square test was used to show relationships between binary or categorical variables. In all tests, results were considered statistically significant if the $\mathrm{p}$ - value was less than 0.05 . A significant chi square test was followed by obtaining the critical value for standardized residual (converted to $\mathrm{Z}$ score). The critical value that corresponds to 0.05 is +1.96 and the variable was considered significant if its critical value was more than 1.96 or less than -1.96 .

\section{RESULTS}

Temporomandibular joint Arthroscopic lysis and lavage was performed to 85 patients $19(22.35 \%)$ males and $66(77.65 \%)$ females with unilateral or bilateral temporomandibular joint internal derangement in Cairo University, Faculty of Dentistry between March 2015 and June 2017. The number of unilateral joint affection was 62 joints and bilateral joint affection was 23 joints with a total number of 108 joints. Age of the selected subjects ranged from 25 to 67 with a mean of 40.3 years.

No perioperative or postoperative major complications occurred; none of the patients developed transient or permanent neurosensory deficits of the facial 
nerve. There were no signs of infection, bleeding, or hematoma. Some patients presented by minimal edema and tenderness at one week with subsequent normal wound healing. They regained their normal ability within one week.

All the patients were included for statistical analysis. According to type of data they were reported as either mean + standard deviations for continuous data or frequencies and percentages for categorical data.

Preoperative MRI diagnosis is shown in table 1, (Fig.2), and (Fig. 3: A,B,C,D,E,F,G)

TABLE (1) Preoperative Diagnoses

\begin{tabular}{|c|c|c|}
\hline Preoperative Diagnosis & Frequency & Percent \\
\hline I & 1 & 0.92 \\
II & 16 & 14.8 \\
III & 33 & 30.56 \\
IV & 24 & 22.22 \\
V & 15 & 13.89 \\
Synovitis & 1 & 0.92 \\
Others* & 18 & 16.67 \\
Total & 108 & 100.0 \\
\hline
\end{tabular}

* Others included: Ankylosing Osteoarthritis, Inflammatory Arthritis, Sublaxation
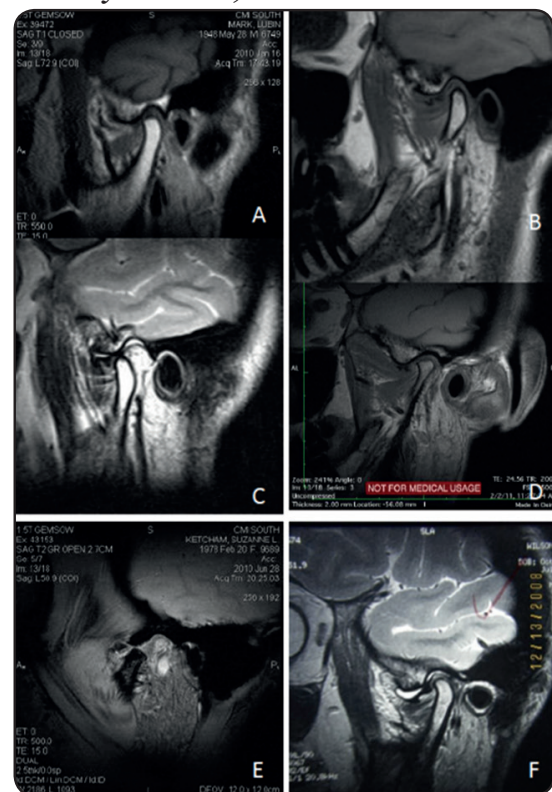

Fig. 2: Abnormal MRI images, showing: A. Wilkes I, B. Wilkes II, C. Wilkes III, D. Wilkes IV, E. Wilkes V, F. Effusion.

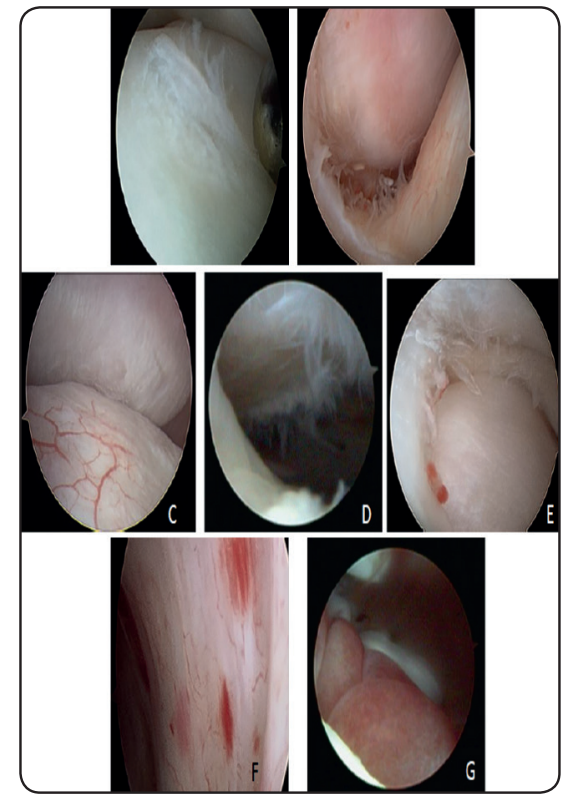

Fig. (3) Abnormal arthroscopic Findings, showing: A. Wilkes I, B. Wilkes II, C. Wilkes III, D. Wilkes IV, E. Wilkes V, F. Synovitis, and G. Others as Inflammatory arthritis)

TABLE (2) Arthroscopic Findings

\begin{tabular}{|c|c|c|}
\hline $\begin{array}{c}\text { Arthroscopic } \\
\text { Findings }\end{array}$ & Frequency & Percent \\
\hline I & 1 & 0.92 \\
II & 14 & 12.96 \\
III & 38 & 35.18 \\
IV & 27 & 25 \\
V & 21 & 19.44 \\
Synovitis & 3 & 2.78 \\
Others* & 4 & 3.7 \\
Total & 108 & 100.0 \\
\hline
\end{tabular}

* Others included: Ankylosing Osteoarthritis, Inflammatory Arthritis, Sublaxation

\section{Correlation between MRI Diagnosis and Ar- throscopic Diagnosis:}

Preoperative diagnosis, based on radiographic assessments, came into co-ordinance with arthroscopic findings in $73(67.51 \%)$ studied joints but were different in the remaining 35 (32.41\%) joints. Ratios are significantly different between the two modalities of diagnosis, $\mathrm{P}=0.001$. Table 3 . 
TABLE (3) MRI diagnosis vs. Arthroscopic findings

\begin{tabular}{|c|c|c|c|}
\hline & Frequency & Percent & P Value \\
\cline { 1 - 3 } Different Diagnosis & 35 & $32.41 \%$ & \multirow{2}{*}{$0.001 *$} \\
\cline { 1 - 2 } Similar Diagnosis & 73 & $67.51 \%$ & \\
\hline
\end{tabular}

Spearman correlation showed a moderate correlation between preoperative MRI diagnosis and arthroscopic findings. $r=0.58, p=0.001$. Table 4
TABLE (4) Correlation between MRI diagnosis and Arthroscopic findings

\begin{tabular}{|l|l|c|c|}
\hline \multicolumn{2}{|l|}{} & $\begin{array}{c}\text { MRI } \\
\text { Diagnosis }\end{array}$ & $\begin{array}{c}\text { Arthroscopic } \\
\text { Findings }\end{array}$ \\
\hline MRI Diagnosis & $\begin{array}{l}\text { Correlation } \\
\text { Coefficient P } \\
\text { value }\end{array}$ & 1.000 & $\begin{array}{c}0.580^{* *} \\
0.001\end{array}$ \\
Arthroscopic & Correlation & $0.580^{* *}$ & 1.000 \\
Findings & Coefficient P & 0.001 & \\
& value & & \\
\hline
\end{tabular}

**Correlation is significant at the 0.01level (2-tailed)

TABLE (5): Accuracy of MRI findings per Wilkes classification

\begin{tabular}{|c|c|c|c|c|c|c|}
\hline & I & II & III & IV & V & P Value \\
\hline Accurate & $76.0 \%$ & $61.4 \%$ & $75.0 \%$ & $76.6 \%$ & $79.7 \%$ & $0.001^{*}$ \\
\hline Z score & $13.5^{\mathrm{n}}$ & $8.9^{*}$ & $6.4^{*}$ & $8.2^{*}$ & $9.6^{*}$ & \\
\hline
\end{tabular}

n Insignificant due to small sample $<5$

* Significant

The accuracy of preoperative MRI diagnosis per Wilkes classification and arthroscopic findings was further assessed using chi square test and $\mathrm{Z}$ score. It showed a statistically significant effect of Wilkes classification on the accuracy of the preoperative clinical and MRI diagnosis when compared to the arthroscopic findings. Table 5

\section{DISCUSSION}

The current study aimed to prove the efficacy and accuracy of TMJ MRI in diagnosing temporomandibular disorders when compared to real arthroscopic findings. We concluded that there is statistically significant difference between the two modalities of diagnosis. It showed also a statistically significant effect of Wilkes classification on the accuracy of the preoperative MRI diagnosis when compared to the arthroscopic findings.
A number of studies were done to compare MRI findings with surgical and autopsy specimens with $90-95 \%$ accuracy $^{3}$. Other studies correlated the clinical findings to MRI findings in accuracy of TMD diagnosis with controversial results ranging from $59 \%$ to $90 \%$, showing inconsistency of MRI diagnosis when compared to RDC/TMD classification $^{22-24}$

Leschied et al showed in their study no significant correlation between MRI and arthroscopic findings of active synovitis, while showing significant difference between joint space width and hyperplastic chronic synovitis. This advocated the use of other modalities as ultrasound for increased joint space, as MRI didn't correlate with acute synovitis spotted with TMJ arthroscopy ${ }^{21}$. 
Going through history; it is established fact that TMJ arthroscopy was discovered and practiced prior to TMJ arthrocentesis by Ohnishi in 1975, with proven efficacy in different TMJ disorders regarding better outcome, Another benefit is allowing accurate diagnosis..$^{25,26}$

Magnetic Resonance Imaging (MRI) scans can be used in conjunction with arthroscopy to correlate with operative findings when indicated. MRI scans used alone for diagnosis show only a two-dimensional picture of the pathologic environment. The practitioner can visualize the "big picture" in the way of displaced disks, joint effusions, and bone degeneration, but fine detail is much better seen with arthroscopy. By getting a visual of the superior joint space, a better diagnosis can be made, a better comprehensive treatment plan can be devised, and ultimately the patient will benefit from use of this scope. Of course, this is being proposed without omitting the fact that primary arthroscopy although being minimally invasive, yet it is still an invasive procedure that need to be indicated for surgical TMJ patients.

Current study showed various limitations, including small sample size, and variation in time duration between performing the MRI and conducting the arthroscopic surgery. Also the effects of the presurgical conservative management on TMJ disease was controlled when MRI was done prior to surgery not initially at time of consultation. The results of current study balanced the specificity of the TMJ MRI with the clinical variations of included patients.

Present study didn't not test or include new MRI modalities like dynamic, contrast enhanced, etc. To follow new studies are required to have larger sample size, more close duration between MRI and arthroscopic procedure, and assessment of new MRI modalities.

Finally current study and other related studies are not denying the fact that the main crucial difference between MRI and arthroscopy is the invasiveness of the procedure, so under no circumstances; arthroscopy can't substitute MRI in being the golden diagnostic tool for TMJ diagnosis if there is no therapeutic indication for the arthroscopic minimally invasive surgical intervention, so the main purpose for those studies would be assessing the sensitivity and specificity of MRI in reaching an accurate TMJ diagnosis in comparison to real life arthroscopic findings.

\section{CONCLUSION}

The primary TMJ arthroscopy procedure has demonstrated an excellent safety and efficacy profile in treating patients with TMJ disorders. The technology has allowed us to establish diagnoses which, otherwise, could not be obtained fully from MRI scans, become more comprehensive with patient care in selecting future procedures based on pathologic findings, and all while lavaging the patient's TMJ to treat acute or chronic symptoms. This study showed significant difference between MRI and Arthroscopic based TMJ diagnosis

\section{REFERENCES}

1. McCain JP SL. Arthroscopy and Arthrocentesis of the And, Temporomandibular Joint. In: Peterson's Principles of Oral Maxillofacial Surgery, Third Edition. ; 2012:49: 1069.

2. Boeddinghaus R, Whyte A. Current concepts in maxillofacial imaging. Eur J Radiol. Published online 2008. doi:10.1016/j.ejrad.2007.11.019

3. Tasaki MM, Westesson PL. Temporomandibular joint: Diagnostic accuracy with sagittal and coronal MR imaging. In: Radiology; 1993. doi:10.1148/radiology.186.3.8430181

4. Katzberg RW, Tallents RH. Normal and abnormal temporomandibular joint disc and posterior attachment as depicted by magnetic resonance imaging in symptomatic and asymptomatic subjects. J Oral Maxillofac Surg. Published online 2005. doi:10.1016/j.joms.2005.04.012

5. Cholitgul W, Nishiyama H, Sasai T, Uchiyama Y, Fuchihata H, Rohlin M. Clinical and magnetic resonance imaging findings in temporomandibular joint disc displacement. Dentomaxillofac Radiol. Published online 1997. doi:10.1038/sj.dmfr.4600239

6. Farina D, Bodin C, Gandolfi S, De Gasperi W, Borghesi A, Maroldi R. TMJ disorders and pain: Assessment by 
contrast-enhanced MRI. Eur J Radiol. Published online 2009. doi:10.1016/j.ejrad.2008.01.014

7. Larheim TA. Role of magnetic resonance imaging in the clinical diagnosis of the temporomandibular joint. In: Cells Tissues Organs. ; 2005. doi:10.1159/000086194

8. Behr M, Held P, Leibrock A, Fellner C, Handel G. Diagnostic potential of pseudo-dynamic MRI (CINE mode) for evaluation of internal derangement of the TMJ. Eur J Radiol. Published online 1996. doi:10.1016/S0720-048X(96)01075-3

9. Conway WF, Hayes CW, Campbell RL. Dynamic magnetic resonance imaging of the temporomandibular joint using FLASH sequences. J Oral Maxillofac Surg. Published online 1988. doi:10.1016/0278-2391(88)90328-X

10. Bell KA, Miller KD, Jones JP. Cine magnetic resonance imaging of the temporomandibular joint. Cranio. Published online 1992. doi:10.1080/08869634.1992.11677928

11. Wang EY, Mulholland TP, Pramanik BK, et al. Dynamic sagittal half-Fourier acquired single-shot turbo spin-echo MR imaging of the temporomandibular joint: Initial experience and comparison with sagittal oblique proton-attenuation images. Am J Neuroradiol. Published online 2007. doi:10.3174/ajnr.A0487

12. Kitai N, Eriksson L, Kreiborg S, Wagner A, Takada K. Three-dimensional reconstruction of TMJ MR images: A technical note and case report. Cranio. Published online 2004. doi:10.1179/crn.2004.010

13. Drace JE, Enzmann DR. Defining the normal temporomandibular joint: Closed-, partially open-, and open-mouth MR imaging of asymptomatic subjects. Radiology. Published online 1990. doi:10.1148/radiology.177.1.2399340

14. Shimazaki Y, Saito K, Matsukawa S, et al. Image quality using dynamic MR imaging of the temporomandibular joint with true-FISP sequence. Magn Reson Med Sci. Published online 2007. doi:10.2463/mrms.6.15

15. Limchaichana N, Petersson A, Rohlin M. The efficacy of magnetic resonance imaging in the diagnosis of degenerative and inflammatory temporomandibular joint disorders: a systematic literature review. Oral Surgery, Oral Med Oral Pathol Oral Radiol Endodontology. Published online 2006. doi:10.1016/j.tripleo.2006.02.001

16. de Leeuw R. Insufficient Evidence for the Efficacy of MRI for Diagnosing TMJ Disorder. J Evid Based Dent Pract. Published online 2008. doi:10.1016/j.jebdp.2007.12.014
17. Shaefer JR, Riley CJ, Caruso P, Keith D. Analysis of criteria for MRI diagnosis of TMJ disc displacement and arthralgia. Int J Dent. Published online 2012. doi: $10.1155 / 2012 / 283163$

18. de Leeuw R. Insufficient Evidence for the Efficacy of MRI for Diagnosing TMJ Disorder. J Evid Based Dent Pract. 2008;8(1):30-32. doi:10.1016/j.jebdp.2007.12.014

19. Schiffman E, Ohrbach R, Truelove E et al. Diagnostic Criteria for Temporomandibular Disorders (DC/TMD) for Clinical and Research Applications: recommendations of the International RDC/TMD Consortium Network* and Orofacial Pain Special Interest Group $\dagger$. J Oral Facial Pain Headache. 2014;28(1):6-27. doi:10.11607/jop.1151

20. Park JW, Song HH, Roh HS, Kim YK, Lee JY. Correlation between clinical diagnosis based on RDC/TMD and MRI findings of TMJ internal derangement. Int J Oral Maxillofac Surg. 2012;41(1):103-108. doi:10.1016/j. ijom.2011.09.010

21. Leschied JR, Smith EA, Baker S, Khalatbari S, Aronovich S. Contrast-enhanced MRI compared to direct joint visualization at arthroscopy in pediatric patients with suspected temporomandibular joint synovitis. Pediatr Radiol. 2019; 49(2):196-202. doi:10.1007/s00247-018-4291-9

22. Barclay P, Hollender LG, Maravilla KR, Truelove EL. Comparison of clinical and magnetic resonance imaging diagnoses in patients with disk displacement in the temporomandibular joint. Oral Surg Oral Med Oral Pathol Oral Radiol Endod. Published online 1999. doi:10.1016/ S1079-2104(99)70191-5

23. Emshoff R, Rudisch A, Innerhofer K, Brandlmaier I, Moschen I, Bertram S. Magnetic resonance imaging findings of internal derangement in temporomandibular joints without a clinical diagnosis of temporomandibular disorder. J Oral Rehabil. Published online 2002. doi:10.1046/ j.1365-2842.2002.00883.x

24. Limchaichana N, Nilsson H, Ekberg EC, Nilner M, Petersson A. Clinical diagnoses and MRI findings in patients with TMD pain. J Oral Rehabil. Published online 2007. doi:10.1111/j.1365-2842.2006.01719.x

25. Zasshi O. Arthroscopy of the temporomandibular joint. Kokubyo Gakkai. 1975;Jun;42((2)):207-213.

26. Ohnishi M. Arthroscopy and arthroscopic surgery of the temporomandibular joint (T.M.J.). Rev Stomatol Chir Maxillofac. Published online 1990. 